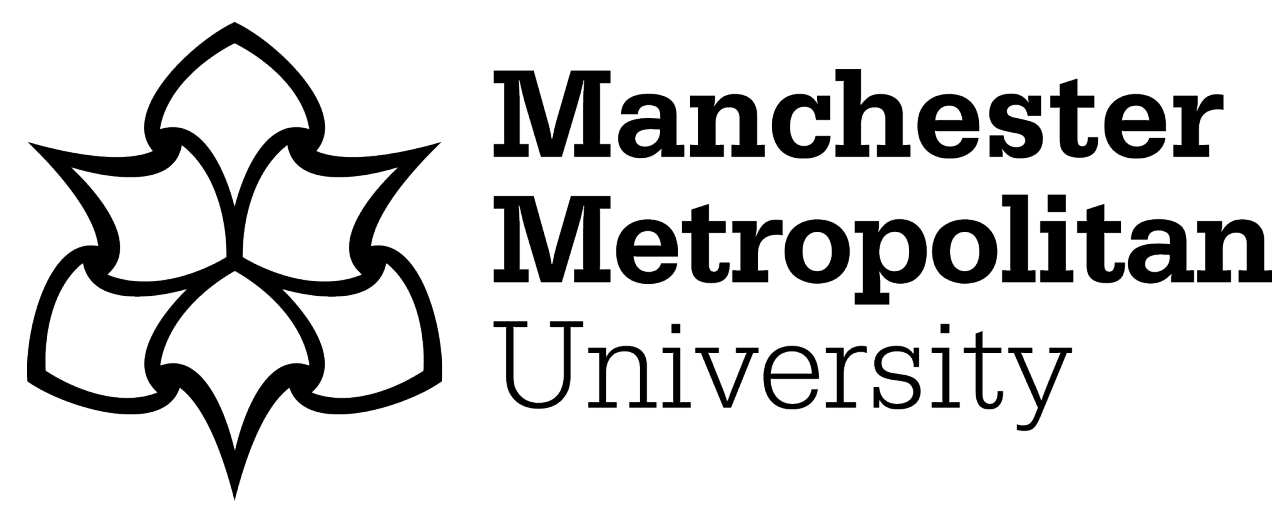

Toorie, AM, Vassoler, FM, Qu, F, Schonhoff, CM, Bradburn, S, Murgatroyd, CA ORCID logoORCID: https://orcid.org/0000-0002-6885-7794, Slonim, DK and Byrnes, EM (2021) A history of opioid exposure in females increases the risk of metabolic disorders in their future male offspring. Addiction Biology, 26 (1). e12856. ISSN 1355-6215

Downloaded from: https://e-space.mmu.ac.uk/624640/

Version: Accepted Version

Publisher: Wiley

DOI: https://doi.org/10.1111/adb.12856

Please cite the published version 


\title{
A history of opioid exposure in females increases the risk of metabolic disorders in their future male offspring
}

\author{
Anika M. Toorie ${ }^{1,4}$ D | Fair M. Vassoler ${ }^{1}$ | Fangfang $\mathbf{Q u}^{2}$ | Christopher M. Schonhoff ${ }^{1}$ | \\ Steven Bradburn ${ }^{3}$ | Christopher A. Murgatroyd ${ }^{3}$ | Donna K. Slonim ${ }^{2}$ | Elizabeth M. Byrnes ${ }^{1}$
}

\author{
${ }^{1}$ Department of Biomedical Sciences, \\ Cummings School of Veterinary Medicine, \\ Tufts University, NorthGrafton, Massachusetts \\ ${ }^{2}$ Department of Computer Science, Tufts \\ University, Medford, Massachusetts \\ ${ }^{3}$ Division of Biomedical Sciences, Manchester \\ Metropolitan University, Manchester, UK \\ ${ }^{4}$ Department of Biology, Rhode Island College, \\ North Providence, Rhode Island \\ Correspondence \\ Elizabeth M. Byrnes, Department of \\ Biomedical Sciences, Cummings School of \\ Veterinary Medicine, Tufts University, \\ Peabody Pavilion, 200 Westboro Road, \\ Grafton, MA 01536. \\ Email: elizabeth.byrnes@tufts.edu \\ Funding information \\ National Institutes of Health, Grant/Award \\ Number: DA025674; Tufts University, Grant/ \\ Award Number: Tufts Collaborates Award
}

\begin{abstract}
Worldwide consumption of opioids remains at historic levels. Preclinical studies report intergenerational effects on the endogenous opioid system of future progeny following preconception morphine exposure. Given the role of endogenous opioids in energy homeostasis, such effects could impact metabolism in the next generation. Thus, we examined diet-induced modifications in F1 male progeny of morphineexposed female rats (MORF1). When fed a high fat-sugar diet (FSD) for 6 weeks, MORF1 males display features of emerging metabolic syndrome; they consume more food, gain more weight, and develop fasting-induced hyperglycemia and hyperinsulinemia. In the hypothalamus, proteins involved in energy homeostasis are modified and RNA sequencing revealed down-regulation of genes associated with neuronal plasticity, coupled with up-regulation of genes associated with immune, inflammatory, and metabolic processes that are specific to FSD-maintained MORF1 males. Thus, limited preconception morphine exposure in female rats increases the risk of metabolic syndrome/type 2 diabetes in the next generation.
\end{abstract}

\section{KEYWORDS}

corticosterone, glucose dyshomeostasis, intergenerational, metabolism, NPY, POMC

\section{I INTRODUCTION}

The percentage of the general population exposed to prescription opioids has increased dramatically over the past decade. ${ }^{1,2}$ Such broadbased opioid exposure has led to millions of people living with opioid use disorders and tens of thousands dying from opioid overdoses. ${ }^{3}$ While such immediate outcomes remain the highest public health priority, additional effects of widespread prescription opioid use within the general population remain unknown. While perhaps not commonly appreciated, in addition to their analgesic and hedonic properties, opioids also produce significant neuroendocrine, immune, and metabolic effects. ${ }^{4}$ These effects are due to the modulatory role that endogenous opioids play in an array of homeostatic processes. ${ }^{5}$ Thus, exposure to exogenous opioids may induce modifications in the endogenous opioid system that impact diverse homeostatic mechanisms beyond those classically associated with opioid addiction.
We previously demonstrated that female opioid exposure in rats, restricted to a 10-day period during adolescence, alters the development of the reproductive axis, delaying sexual maturation and resulting in transcriptional modifications in the hypothalamus that persist for months after this discrete exposure period. ${ }^{6}$ Moreover, we observe significant sex-specific effects in the progeny of adolescent morphine-exposed females, despite the fact that females are drug free for several weeks prior to mating. Our initial studies primarily focused on effects related to drug sensitivity with a primary emphasis on reward systems. These studies revealed significant alterations in both the F1 and F2 generation, ${ }^{6-11}$ supporting the hypothesis that adolescent morphine exposure can trigger transgenerational effects maintained for at least two generations.

In these previous studies, altered opioid-mediated regulation of homeostatic processes regulated within the hypothalamus were observed and those effects persisted for two generations in male, 
but not female, offspring of morphine-exposed females (MORF1 males). ${ }^{12}$ Additional studies found that proopioimelanocortin (Pomc) gene expression was up-regulated in both adolescent and adult MORF1 males, but not MORF1 females. ${ }^{10,13}$ The hypothalamic opiomelanocortin system originates in the arcuate nucleus (ARC), where several neuropeptides involved in the regulation of sexual maturation, bodyweight, and metabolism are produced. ${ }^{14-16}$ Previous findings in both animals and humans suggest that changes in the regulation of POMC neurons may play an important role in transgenerational epigenetic effects associated with parental nutrition. ${ }^{17}$ Thus, given the role of POMC as a critical regulator of metabolic homeostasis and the transgenerational effect on Pomc expression observed in MORF1 males, we hypothesized that discrete exposure to morphine prior to conception will alter hypothalamic regulation of metabolic processes in future male offspring.

To test this hypothesis, we examined F1 male offspring maintained on a diet containing increased fat and sugar as compared with those on a standard control diet (CD). Endpoints included the timing of sexual maturation, body weight gain, feeding, and locomotion. We next examined fasting blood glucose levels and plasma concentrations of insulin and corticosterone (CORT), which are both important glucoregulatory factors. ${ }^{18}$ Based on significant F1 differences, we also examined expression of ARC POMC and the orexigenic polypeptide neuropeptide $Y$ (NPY), as well as ARC POMC-derived $a-M S H$ and $\beta$ EP concentration in select target sites. Finally, to further elucidate likely mechanisms behind the observed differences, the ARC transcriptome was analyzed to identify functional pathways in F1 males modified by maternal opioid history in a diet-specific manner. Collectively, our findings demonstrate that opioid exposure in females, even when that exposure occurs prior to conception, increases the risk of diet-induced type 2 diabetes in their adult male offspring and that these effects are associated with significant alterations in transcriptional pathways that regulate metabolic, immune, and neurodevelopmental processes within the ARC.

\section{2 | MATERIALS AND METHODS}

\section{1 | Experimental subjects}

\subsection{1 | Adolescent morphine exposure}

For all experiments, 22-day-old female Sprague-Dawley rats [Crl:CD (SD)BR] were purchased from Charles River Breeding Laboratories and grouped housed in light (on 600-1800 hours) and temperature $\left(21^{\circ} \mathrm{C}-24^{\circ} \mathrm{C}\right)$ regulated rooms with ad libitum access to food and water. Adolescent rats were administered morphine (MORFO; $\mathrm{n}=$ 36) or saline (SALFO; $n=36$ ) beginning on postnatal day 30 (PND30) for 10 days, which corresponds to the early-midadolescent phase in humans. ${ }^{19}$ Morphine was administered daily between 9:00 and 10:00 AM using an intermittent, increasing dose regimen of morphine that increased every other day $(5,10,15,20$, and $25 \mathrm{mg} / \mathrm{kg}$; sc).
SALFO females were administered saline at equivalent volumes. This study was performed in three independent cohorts.

\subsection{2 | Mating}

All FO females remained drug free for at least 3 weeks after the final morphine injection and were subsequently mated as adults (after PND60) with drug-naive colony males. On the day following parturition (PND1), litters were culled to five males and five females housed together until weaning. On PND21 litters were weaned, housed with same sex littermates, and transitioned to standard rodent chow. Given our previous findings indicating that male but not female MORF1 animals demonstrate significant differences in POMC gene expression and hypothalamic dysregulation in response to exogenous opioids, these studies were conducted in male offspring. For every experimental group, only one male from each litter was used to limit potential litter effects.

\section{2 | Experimental procedures}

\subsection{1 | Diets and bodyweights}

On PND30, MORF1 and SALF1 males were transitioned to a CD (3.8 $\mathrm{kcal} / \mathrm{gm}$ ) consisting of $10 \%$ fat/0 \% sucrose (D12450K; Research diets, New Brunswick, NJ) or a high fat-moderate sucrose diet (fatsugar diet [FSD]) $(4.7 \mathrm{kcal} / \mathrm{gm}$ ) consisting of $45 \% \mathrm{fat} / 17 \%$ sucrose diet (D12451; Research diets) for a period of 6 weeks. Individual bodyweights (grams) were taken weekly throughout the study with bodyweight gain calculated as PND72-PND30.

\subsection{2 | Sexual maturation}

The onset of puberty was measured using preputial separation. ${ }^{20}$ Beginning on PND30, subjects were examined once daily for skin-fold separation via gentle retraction of the prepuce membrane with sexual maturation defined as the first date of complete preputial retraction.

\subsection{3 | Caloric consumption}

Grouped house animals were fed their respective diets for 6 weeks, after which individual animals were transferred to a clean cage with $300 \mathrm{~g}$ of food provided. Twenty-four hours later, the remaining food, including any pellets on the cage floor, was weighed and caloric consumption was calculated by subtracting final food weight from initial food weight multiplied by $3.8 \mathrm{kcal}$ for $C D$ and $4.7 \mathrm{kcal}$ for FSD.

\subsection{4 | Locomotor activity}

On PND72 home cages were individually placed into activity frames at 10:00 AM. Following a 2-hour acclimation period activity was monitored for 24 hours using the SmartFrame Motor Monitor System 
(Hamilton-Kinder; Poway, CA), with free access to food and water throughout. Activity data were analyzed as distance travelled $(\mathrm{cm})$ based on sequential photobeam breaks.

\subsection{5 | Blood and brain collections}

Between PND70-72, all animals were fasted overnight (16 hours). Between 9:00 and 10:00 AM, animals were transferred to a procedure room (transit $<60$ seconds), exposed briefly to $\mathrm{CO}_{2}$, and decapitated. Blood glucose levels were measured immediately from trunk blood using a standard glucometer (CVS, MA) with remaining trunk blood collected into heparinized tubes. Brains were removed, rapidly frozen in $-20^{\circ} \mathrm{C}$ in 2 -methylbutane, and stored at $-80^{\circ} \mathrm{C}$. Heparinized blood was centrifuged, and plasma was stored at $-20^{\circ} \mathrm{C}$. For protein and peptide analysis, brains were cryostat-mounted and 1-mm ${ }^{3}$ bilateral micropunches were taken from the ARC (A/P -2.3; M/L \pm 1 ; V/D -8 $\mathrm{mm}), \mathrm{PVN}(\mathrm{A} / \mathrm{P}-1.3 ; \mathrm{M} / \mathrm{L} \pm 0.5 ; \mathrm{V} / \mathrm{D}-6.8 \mathrm{~mm})$, and VTA (A/P -4.7; $\mathrm{M} / \mathrm{L} \pm 1.5$; V/D $-8.2 \mathrm{~mm}$; all coordinates relative to Bregma).

\subsection{6 | Western blotting}

Tissue punches were homogenized in Pierce immunoprecipitation lysis buffer (Thermo Fisher Scientific, Waltham, MA) supplemented with 1× proteinase inhibitor cocktail and $1 \mu \mathrm{M}$ PMSF, and total protein content quantified via Pierce BCA protein assay (Thermo Fisher) according to manufacturer's recommendations. Total protein $(20 \mu \mathrm{g})$ was separated in a $4 \%$ to $12 \%$ Tris-Bis gel (NuPage Novex, Thermo Fisher) and transferred to a nitrocellulose membrane (Thermo Fisher). Membranes were washed, blocked in $5 \%$ bovine serum albumin (BSA) or nonfat milk for 1 hour, and incubated overnight at $4^{\circ} \mathrm{C}$ in primary antibodies recognizing either NPY (Cell Signaling, D7Y5A XP; Danvers MA), POMC (Abcam, ab180766; Cambridge, MA), or $\beta$-actin (Abcam, ab6721). The next day, membranes were washed and incubated in the appropriate HRP-secondary antibody (2 hours at room temperature), enhanced with chemiluminescence solution (Pierce ECL Western Blotting substrate; Thermo Fisher scientific), and imaged (BioRad Universal Hood II ChemiDoc XRS) with densitometry determined in ImageJ (NIH; Bethesda, MD). Antibody specificity was probed using purified peptide (Recombinant Human POMC protein Ab108118 and NPY endogenous neuropeptide Ab120208). Both were run in isolation and in arcuate lysates spiked with the peptide. In addition, both dilution curves and negative controls were utilized.

\subsection{7 | Enzyme immunosorbent assays}

Quantification of a-MSH (EK04301; Phoenix Pharmaceuticals; Burlingame, CA), $\beta$-EP (EK02233; Phoenix Pharmaceuticals), insulin (Crystal Chem, 90060; Downers Grove, IL), and corticosterone (Abcam; ab108821) was performed using commercially available EIA kits. Assays were performed according to the manufacturer's suggestions. Intra-assay and interassay CV of both a-MSH and $\beta$-EP EIA's are $<10 \%$ and $<15 \%, 5.3 \%$ and $10.6 \%$ for corticosterone EIA, and $<10 \%$ and $<10 \%$ for insulin EIA

\subsection{Arcuate nucleus RNA sequencing}

A separate cohort of SALF1 and MORF1 animals were generated, maintained on the respective diets for 6 weeks, overnight fasted, and euthanized the following day. Bilateral ARC tissue was collected as described above ( $N=6$ /group ). Total RNA was extracted using the Qiazol RNA extraction kit (Qiagen) and $2 \mu \mathrm{g}$ of total RNA was purified for mRNA. Isolated mRNA was further subjected to fragmentation, cDNA synthesis, adapter ligation, purification, and enrichment reactions (Illumina TruSeq Stranded mRNA sample preparation kit; Illumina) to generate an equimolar cDNA library; 51-bp single-end reads were generated on an Illumina HiSeq2500 Sequencer at the Tufts University Core Facilities (TUCF) Genomics Core (Boston, MA, USA).

\subsection{1 | RNA-seq mapping and normalization}

Reads were aligned to the reference genome Rattus norvegicus 6.0 using the STAR alignment software, v2.5.3a. ${ }^{21}$ Aligned reads were counted against gene model annotation (Ensembl v92) to obtain expression values using the STAR gene-counts option. The three samples with fewer than 10 million reads uniquely aligned to the annotated transcriptome were discarded. Read counts were normalized using the trimmed mean of $M$ values (TMM) normalization method. ${ }^{22}$

\section{4 | Statistical analyses}

\subsection{1 | Behavioral and physiological endpoints}

The results for each treatment are presented as mean \pm SEM. Most data sets were analyzed using a two-way analysis of variance (ANOVA) with maternal opioid history and diet as factors with post hoc effects examined using Holms-Sidak multiple comparisons test. Even in the absence of significant interactions, planned comparisons regarding the effects of maternal treatment within diet condition were conducted. ${ }^{23} \beta$-EP data between diet conditions violated normality that could not be eliminated via transformation. Thus, these data were analyzed using two-tailed $t$ tests within each dietary condition. For nonparametric data, a Mann-Whitney $U$ sum of ranks test was performed, while two-tailed $t$ tests with Welch correction were performed for data sets displaying unequal variances. For data sets analyzed nonparametrically, graphs are presented using mean \pm SEM for continuity of data presentation. Preputial separation data were analyzed using a chi-square test on the percent of subjects displaying initial preputial separation by day. For all analyses, significance was defined as $P<.05$.

\subsection{2 | RNA sequencing}

Genes with a count of at least one in at least six samples, and a count of at least 10 in at least one sample, were retained for differential expression analysis. The EdgeR generalized linear model was used to analyze differential expression. ${ }^{24}$ Differentially expressed genes were 
identified using an adjusted false discovery rate (FDR) $q$ value $<0.05$. Functional enrichment of Gene Ontology Biological Process terms in the sets of differentially expressed genes was identified using the ClusterProfiler R package. ${ }^{25}$ Gene set enrichment analysis (GSEA) was further applied for pairwise comparisons between classes, using the curated gene sets (MSigDB c2) and the transcription factor target gene sets (c3) (http://www.broadinstitute.org/gsea/index.jsp). ${ }^{26}$ Significance of the enrichment under GSEA was assessed based on 1000 gene set permutations and was FDR adjusted. We reported all gene sets including between five and 500 genes with FDR $<0.1$. Molecular interactions shown in Figure 5D reflect either MSigDB c2 TF-target relationships or protein-protein interactions from the STRING database. ${ }^{27}$ We used only interactions derived from experimental evidence, with confidence score $\geq 400$, in rat or transferred from orthologs in other organisms.

\section{3 | RESULTS}

Using an established model of discrete female adolescent morphine exposure, we determined the impact of preconception opioid exposure on metabolic-related processes in adult male offspring (see Figure $1 \mathrm{~A}$ ). It is important to note that no differences in fertility or fecundity, litter size, sex distribution, birth weight, postnatal weight gain, or timing of postnatal developmental milestones (tooth eruption or eye opening) have been observed in this phenotype. ${ }^{7,10}$ As demonstrated in Figure 1B, however, preputial separation data revealed delayed sexual maturation in MORF1 males irrespective of diet $\left(\left[x^{2}\right.\right.$ $(1, \mathrm{~N}=99)=14.97, P<.001 ; \mathrm{PND} 39]$ and $\left[X^{2}(1, \mathrm{~N}=99)=7.64, P\right.$ $=.006$; PND40]). As shown in Figure $1 \mathrm{C}$, there were significant main effects of both maternal opioid history and diet $\left(F_{1,95}=5.38 ; P<.03\right.$ and $F_{1,95}=17.47 ; P<.0001$, respectively) with a trend toward an

(A) Experimental Design

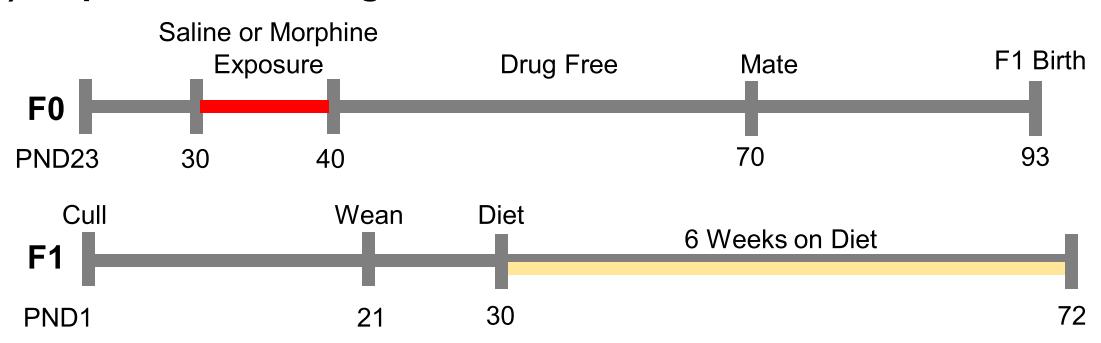

(B) Preputial Separation

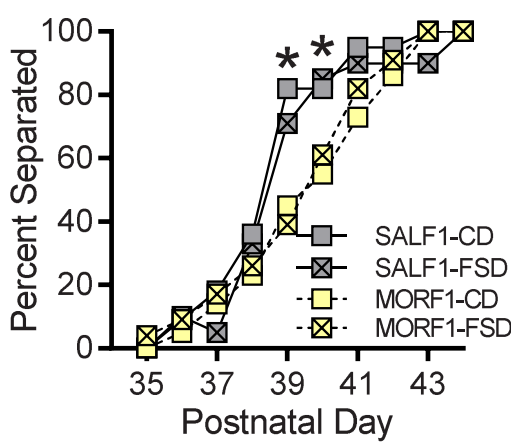

(C) Bodyweight Gain

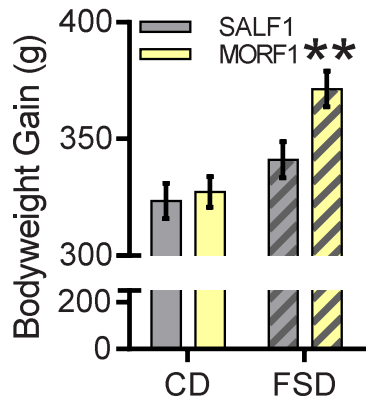

(E) Locomotor Activity Following 6-Weeks On Diet

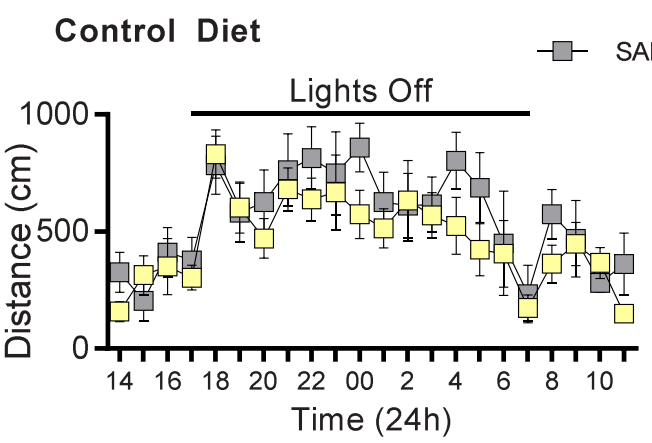

(D) Food Consumption

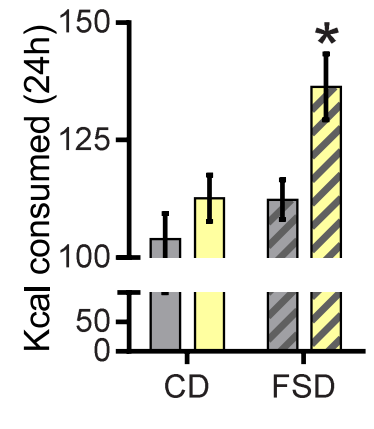

Fat Sugar Diet

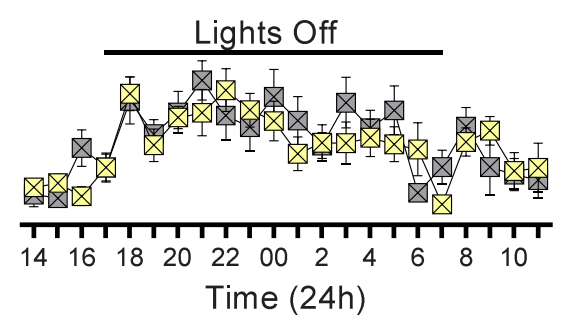

FIGURE 1 Sexual development and energy balance profile. A, Outline of experimental design. B, Percent separated F1 animals at postnatal days 35-44. MORF1's displayed delayed indices separation as compared with SALF1's. C, Mean \pm SEM, bodyweight gain between PND30 and PDN72 following 6-wk control diet (CD) or high fat-sugar diet (FSD). FSD-MORF1s displayed elevated bodyweight compared with SALF1 males, whereas F1s fed a control diet gain comparable weight. ${ }^{*} P<.05, N=24-25$. D, Mean $\pm S E M, 24$-h caloric consumption was assessed in post 6-wk diet maintenance, $\mathrm{N}=11-12$. FSD-MORF1s consumed more food than FSD-SALF1s, while F1s consumed similar amounts when fed a CD. E, Mean \pm SEM, locomotor activity in MORF1 and SALF1s assessed following CD or FSD. Main effect of time across diet conditions. ${ }^{*} P<.05, \mathrm{~N}=9 / \mathrm{group}$ 
interaction ( $F_{1,95}=3.2 ; P=.08$ ) on body weight gain between the start of the diet and the day of sacrifice (PND30-PND72). Planned comparisons revealed that MORF1 males gained significantly more weight in comparison with SALF1 males when maintained on a diet containing elevated levels of fat and sugar $\left(t_{1,95}=2.95 ; P<.01\right)$. Additionally, when body weight data were examined overtime, there was a significant main effect of time $\left(F_{7,588}=7889, P<.001\right)$ as well as maternal opioid history $\left(F_{7,588}=4.55, P<.001\right)$, time by diet $\left(F_{7,588}=10.55\right.$, $P<.001)$ and time by maternal opioid history $F_{7,588}=3.03, P<.01$ (see Data S1; Figure 1).

As changes in bodyweight could be due in part to changes in feeding behavior or activity levels, F1 males were examined for changes in these parameters. Caloric intake monitored over 24 hours revealed significant main effects of both maternal opioid history and diet $\left(F_{1,41}=8.80, P<.006 ; F_{1,41}=8.45 ; P<.007\right.$, respectively), with no significant interaction $\left(F_{1,41}=1.92 ; P=.17\right.$; Figure $\left.1 D\right)$. Planned comparisons indicated that FSD-fed MORF1 males consumed more calories than FSD-fed SALF1 males $\left(t_{1,41}=3.05 ; P<.01\right)$. In contrast, as represented in Figure 1E, locomotor activity followed the expected circadian rhythm with a main effect of time observed under each dietary condition $\left(F_{23,368}=6.93, P<.001[C D]\right.$ and $F_{23,368}=7.22, P$ $<.001$ [FSD]). However, there were no main effects of maternal opioid history under either diet condition $\left(F_{1,16}=2.67, P=.12[C D]\right.$ and $\left.F_{1,16}=0.45, P=.51[F S D]\right)$ and no time by maternal opioid history interaction $\left(F_{23,368}=0.59, P=.94[C D]\right.$ and $F_{23,368}=0.98, P=.48$ [FSD]).

Plasma from fasted SALF1 and MORF1 males was examined for potential alterations in glucose homeostasis. As shown in Figure 2A, there were significant main effects of maternal opioid history and diet as well as a significant interaction $\left(F_{1,40}=6.79 ; P<.02 ; F_{1,40}=4.33 ; P\right.$ $<.05$ and $F_{1,40}=4.52 ; P<.04$, respectively). Post hoc analysis revealed significantly elevated fasting glucose in MORF1 relative to SALF1 males following FSD $\left(t_{1,40}=3.425 ; P<.01\right)$. We next tested levels of insulin, as a potential mechanism mediating the hyperglycemia displayed in the MORF1 group. As depicted in Figure 2B, there were no significant main effects of maternal opioid history or diet condition
$\left(F_{1,62}=0.04, P=.85 ; F_{1,62}=0.25, P=.62\right.$, respectively); however, there was a significant interaction $\left(F_{1,62}=5.99, P<.02\right)$, with post hoc analysis revealing elevated insulin levels in FSD-maintained MORF1 males compared with FSD-maintained SALF1 males $\left(t_{1,62}=\right.$ $2.20, P<.05)$. Finally, as demonstrated in Figure $2 \mathrm{C}$, there was a main effect of maternal opioid history $\left(F_{1,43}=5.03 ; P<.04\right)$ on plasma CORT levels. There was no main effect of diet and no interaction ( $F_{1,43}=0.71, P=.41 ; F_{1,43}=1.27, P=.27$, respectively); however, post hoc analyses revealed that MORF1 males had significantly elevated CORT in comparison with SALF1 males only under FSD conditions $\left(t_{1,43}=2.37 ; P<.05\right)$.

As central regulators of energy homeostasis, NPY and POMC polypeptides in the ARC were measured to examine their roles in mediating bodyweight/feeding behavior and glucose regulation in MORF1 male progeny. We assessed both proNPY (precursor to the bioactive NPY peptide) and NPY levels. As shown in Figure 3A, there was a significant effect of maternal opioid history on proNPY expression $\left(F_{1,44}\right.$ $=7.48 ; P<.01)$ with no effect of diet and no interaction $\left(F_{1,44}=0.14\right.$; $\left.P=.07 ; F_{1,44}=0.35 ; P=.56\right)$. Post hoc analysis revealed that MORF1 males under $C D$ conditions displayed significantly more proNPY $\left(t_{1,44}\right.$ $=2.30 ; P<.05$ ). With regard to NPY levels (Figure 3B), there was a trend toward a main effect of maternal opioid history, a main effect of diet, and a significant maternal opioid history by diet interaction $\left(F_{1,46}=3.65 ; P=.06 ; F_{1,46}=9.06 ; P<.005 ; F_{1,46}=4.15 ; P<.05\right.$, respectively). Post hoc analysis indicated that MORF1 males exhibit significantly increased levels of NPY when compared with SALF1 males under FSD conditions ( $\left.t_{1,46}=2.73 ; P<.05\right)$.

As shown in Figure $4 \mathrm{~A}$, POMC levels in the ARC revealed no significant main effects of maternal opioid history or diet $\left(F_{1,46}=3.21, P\right.$ $=.08 ; F_{1,46}=1.09 ; P=.30$, respectively). There was, however, a significant maternal opioid history by diet interaction $\left(F_{1,46}=6.34 ; P<.02\right)$, with post hoc analysis revealing significantly higher levels of POMC within the ARC of MORF1 males relative to SALF1 males under CD conditions $\left(t_{1,40}=2.99 ; P<.01\right)$. As we have previously observed significantly increased Pomc expression in MORF1 males, ${ }^{10}$ we next determined whether increased POMC levels under standard diet
(A) Fasting Glucose

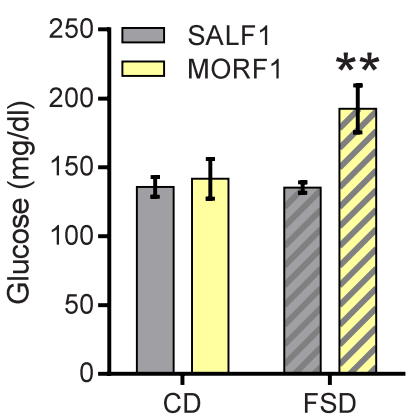

(B) Fasting Insulin

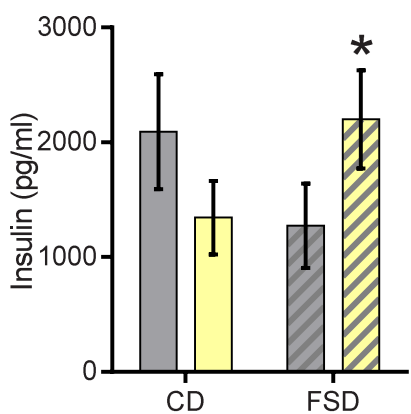

(C) Fasting Corticosterone

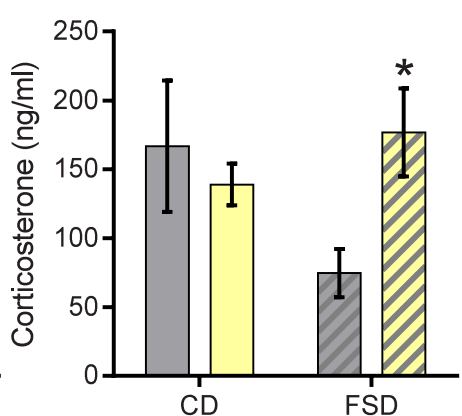

FIGURE 2 Fasting glucose, insulin, and corticosterone levels. A, Mean \pm SEM, fasting glucose levels (mg/dL). Following a 16-h fast, glucose levels were measured. Fat-sugar diet (FSD)-MORF1s exhibit elevated fasting glucose whereas control diet (CD)-F1s display comparable levels. ${ }^{*} P<.05$, ${ }^{* *} P<.01, \mathrm{~N}=10-12$. B, Mean $\pm \mathrm{SEM}$, plasma insulin levels were assessed $(\mathrm{pg} / \mathrm{mL})$. FSD-fed MORF1s exhibit elevated fasting insulin while CD-fed F1s exhibit similar plasma insulin. ${ }^{*} P<.05, \mathrm{~N}=13-18$. C, Mean $\pm \mathrm{SEM}$, fasting corticosterone levels ( $\mathrm{ng} / \mathrm{mL}$ ). FSD-MORF1s exhibit elevated corticosterone compared with FSD-SALF1s while CD-fed F1s display comparable levels of plasma corticosterone. ${ }^{*} P<.05, N=10-14$ 


\section{(A) proNPY in the Arcuate}

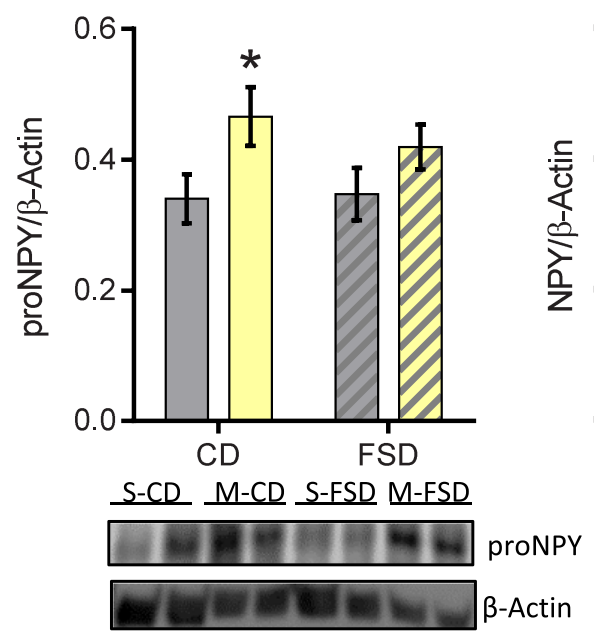

(A) POMC in the Arcuate

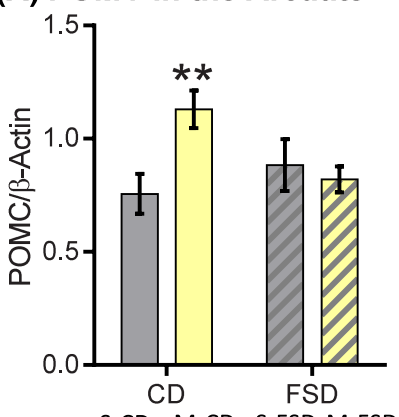

S-CD M-CD S-FSD M-FSD

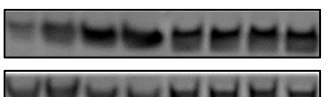

(B) NPY in the Arcuate

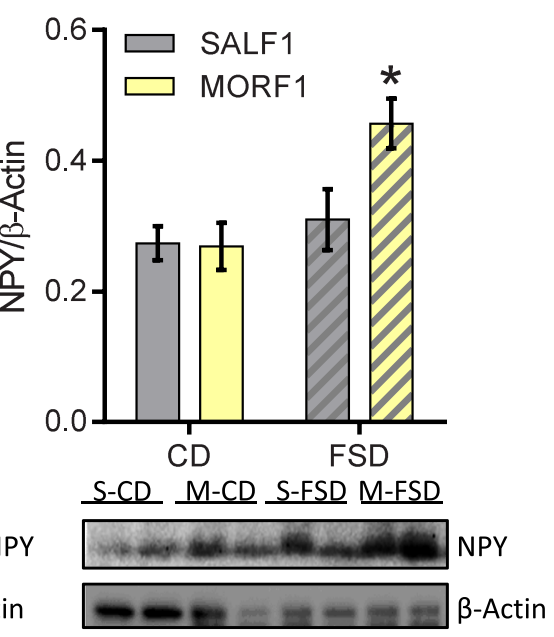

FIGURE 3 ARC proNPY and NPY levels. A, Mean \pm SEM, proNPY relative to $\beta$-actin. Control diet (CD)- and FSD-fed MORF1s display increased proNPY polypeptide. B, NPY relative to $\beta$-actin. Fat-sugar diet (FSD)-fed MORF1 males display increased NPY, whereas similar levels are observed between F1s fed a CD. ${ }^{*} P<.05, N=11-14$
(B) $\beta$-EP in the VTA

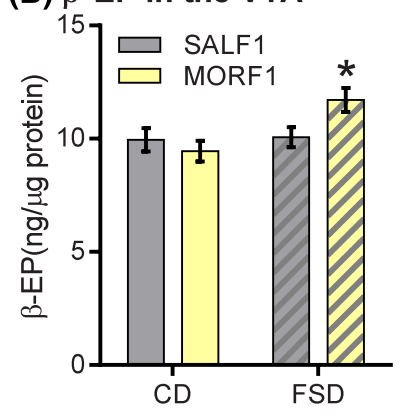

\section{(C) $\alpha-M S H$ in the PVN}

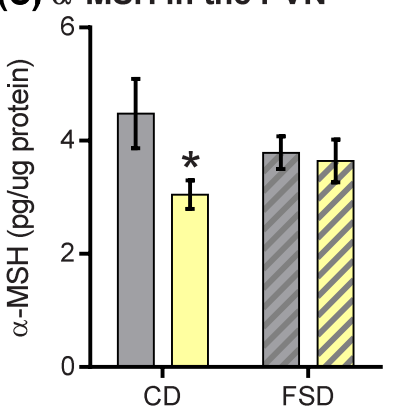

FIGURE 4 POMC, MSH, and $\beta$-EP levels in F1 hypothalamus and midbrain. A: Mean \pm SEM, ARC POMC relative to $\beta$-actin. MORF1 males display elevated POMC relative to SALF1 on a control diet, ${ }^{*} P<.05, \mathrm{~N}=8-10$. B, Mean \pm SEM, a-MSH in PVN. Control diet-fed MORF1 males display reduced $a-M S H$ levels in PVN, ${ }^{*} P<.05, N=7$. D: Mean \pm SEM, $a-M S H$ in the VTA. Fat-sugar diet (FSD)-fed MORF1s displayed elevated $\beta$-EP compared with SALF1s, ${ }^{*} P<.05, \mathrm{~N}=7-11$

conditions might relate to epigenetic programming of Pomc regulation. Thus, we performed DNA methylation analysis across the rat Pomc locus. However, this revealed no significant differences within the promoter, exon 2 or exon 3 CpG islands between SALF1 and MORF1 males (Data S1).

As we observed differences in POMC levels under specific diet conditions based on maternal opioid history, we next examined levels of peptides cleaved from POMC known to regulate energy balance. Namely, $a-M S H$ and $\beta$-EP peptides were measured within the PVN and VTA where they are known to facilitate changes in feeding behaviors. In the VTA, there was no effect of maternal drug history on $\beta$-EP under CD conditions (Mann U: 29; $P<.21$ ); however, there was a significant effect of maternal drug history within FSD conditions $\left(t_{1,17}=\right.$ 2.37; $P=.03$ ), with MORF1 males displaying higher levels of $\beta$-EP (Figure 4B). In contrast, there were no effects of maternal morphine history or diet and no interaction on a-MSH levels within the VTA $\left(F_{1,35}=00992, P=.75 ; F_{1,35}=2.33, P=.14 ; F_{1,35}=0.0009, P=.98\right.$, respectively; Data S1; Figure 2). However, within the PVN, there was a significant main effect of maternal drug history on a-MSH levels, with no effects of diet or maternal drug history by diet interaction (Figure 4C; $F_{1,34}=4.29, P<.05 ; F_{1,34}=0.006, P=.94 ; F_{1,34}=$ 2.13; $P=.15$, respectively).

Given that the significant effects of maternal opioid history on F1 bodyweight gain, feeding behavior, and glucose regulation were associated with diet-specific alterations in neuronal populations within the ARC critical for the regulation of energy homeostasis (POMC and NPY), we wanted to further elucidate potential mechanisms underlying these alterations. We therefore performed RNA sequencing of ARC tissue in a new cohort of animals to identify molecular pathways affected by maternal opioid history and diet. All animals were examined following a 16-hour (overnight) fast to correspond to data showing glucose dysregulation. Expression data were initially analyzed using EdgeR to identify individual differentially expressed genes via a multifactorial analysis, with maternal opioid history and diet as factors. This analysis yielded 168 significantly differentially expressed genes between MORF1 and SALF1 FSD-maintained males (see Figure 5A). 
(A) Volcano Plot and Heatmap of 168 Top Gene Changes SALF1 and MORF1 FSD

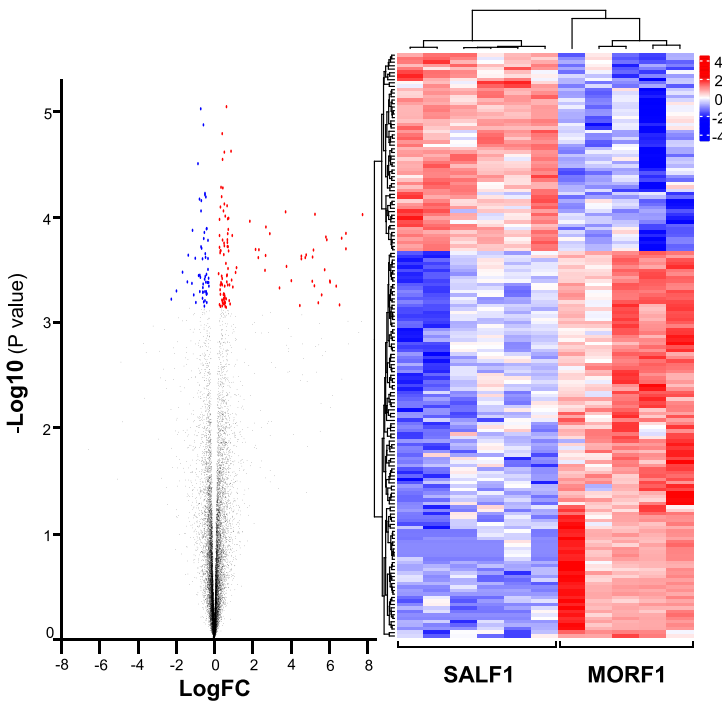

(C) GO Terms Enriched in Up-Regulated DEG SALF1 and MORF1 FSD

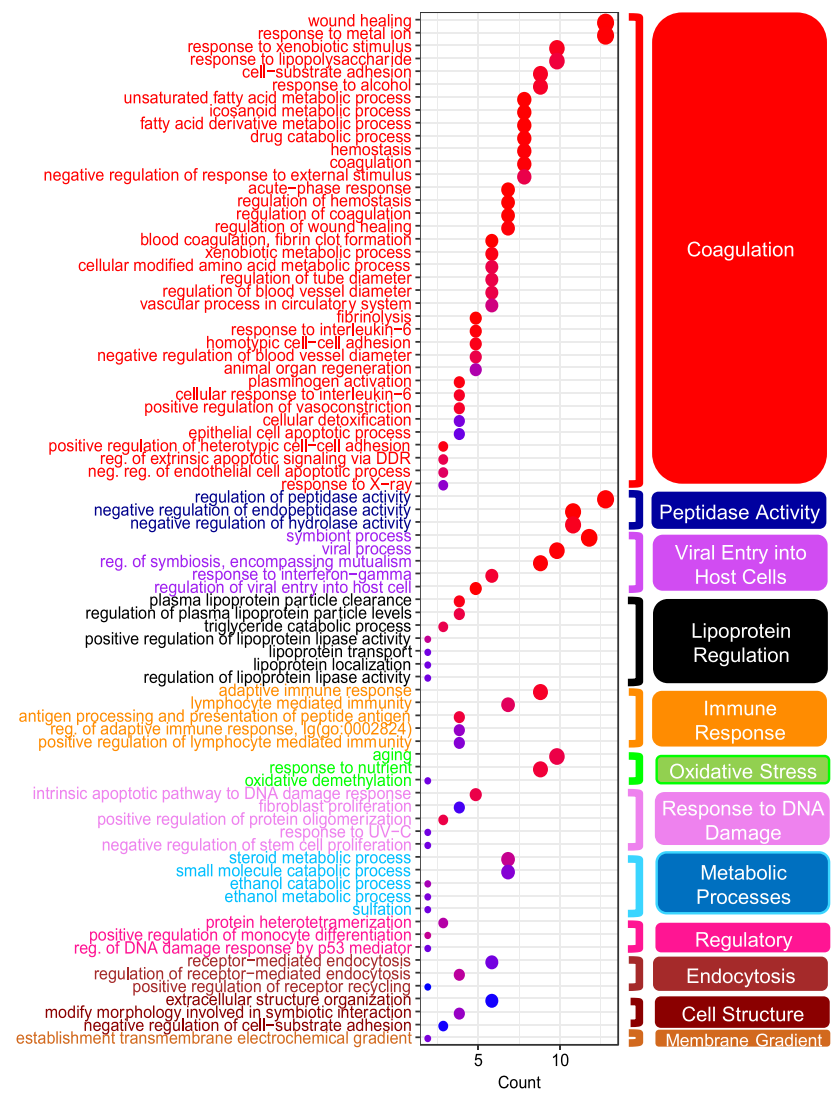

(B) GO Terms Enriched in Down-Regulated DEG SALF1 and MORF1 FSD

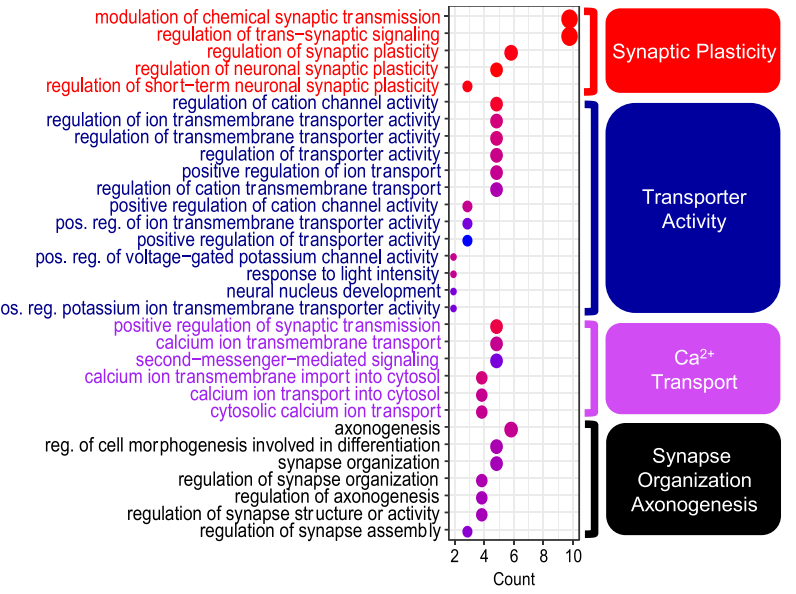

(D) Transcription Factors with Leading Edge Targets

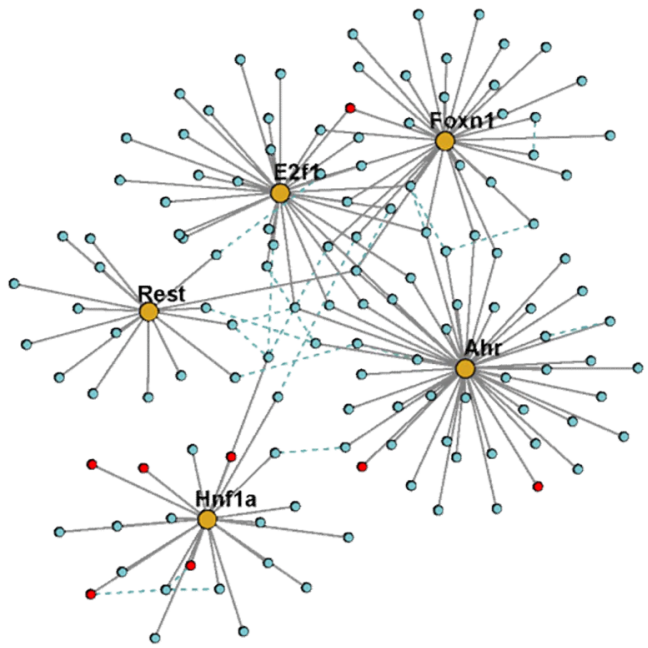

FIGURE 5 RNA-seq transcriptome analysis from the arcuate nucleus. All comparisons are between SALF1 and MORF1 maintained on fat-sugar $\operatorname{diet}(F S D)$. A, A volcano plot presenting all expressed genes ( $q$ value < 0.05). Blue = down-regulated; Red = up-regulated. Those 168 differentially expressed genes (DEGs) with regularized-logarithm transformed count value are shown in the adjacent heat map. The two groups of rats (SALF1 and MORF1) were separated using hierarchical clustering. Gene Ontology (GO) terms enriched in down-regulated B, and up-regulated C, DEGs. Hierarchical clustering identified functional groups based on similarity of differential genes in each term and are organized by color. D,

Transcription factors (gold) and their leading-edge targets (ie, genes contributing most to the TFs' significance) from the GSEA TF-target analysis. Red circles indicate significant DEGs. Dashed lines indicate protein-protein interactions between the target genes (see Section 2)

Of these 168 genes, 119 were significantly up-regulated in MORF1, and 57 were down-regulated. No significantly differentially expressed individual genes were identified in any of the other comparisons (eg, MORF1 vs SALF1 CD-maintained, FSD vs CD either with or without 
maternal opioid history, or main effects of diet and maternal opioid history).

We analyzed Gene Ontology (GO) enrichment in this set of 168 differentially expressed genes using ClusterProfiler ${ }^{25}$ to obtain a comprehensive understanding of the overrepresented functional categories. Genes down-regulated with morphine in the FSD-maintained animals were enriched for $31 \mathrm{GO}$ biological process terms (FDR $p$ adjust <.05; see Figure 5B). Modulation of chemical synaptic transmission was the most significantly enriched biological process among these genes. Based on the similarity of the differentially expressed genes in each term, the enriched pathways in genes down-regulated in MORF1 males were further clustered into four major categories that can broadly be described as synaptic plasticity, transporter activity, calcium ion transport, and synapse organization and axonogenesis (Figure 5B). These categories are consistent with the suggestion that molecular mechanisms of neuronal signaling in the ARC of MORF1 males are impaired when those males are maintained on a FSD. In contrast, the set of differentially expressed genes up-regulated in MORF1 males was enriched for $80 \mathrm{GO}$ biological process terms (after removing highly similar and redundant terms). These clustered into several broad categories, including coagulation, fatty acid and xenobiotic metabolic processes, response to interleukin-6, peptidase activity, plasma lipoprotein particle clearance, and viral entry into host cells (Figure 5 C).

GSEA $^{26}$ differs from the above analyses because genes that do not change enough to appear significant at an individual gene level may nonetheless show a significant pattern as part of a coordinately functioning gene set. Full GSEA results appear in Data S2. Applying GSEA with the "c2 canonical pathways" gene sets from MSigDB ${ }^{26}$ revealed a significant induction of complement and coagulation cascades, ECM regulators, cytokine-cytokine receptor interaction, and drug metabolism/cytochrome p450 in MORF1 FSD-maintained males. Interestingly, the PPAR signaling pathway was found among the top significant up-regulated pathways. Moreover, pathways involved in neuronal systems, voltage gated potassium channels, and amine ligand binding receptors were significantly repressed in MORF1 FSD-maintained males.

Further, to attempt to identify regulators of the observed gene expression dynamics between FSD-maintained MORF1 and SALF1 males, we performed GSEA with gene set collections corresponding to transcription factor targets (part of the c3 collection in MSigDB) or those genes affected by various genetic and chemical perturbations in prior experiments (part of the c2 collection). Hnf1 was the only transcription factor whose targets were significantly up-regulated in MORF1 males, while Rest, Foxn1, Ahr, and E2F1 targets were significantly down-regulated in MORF1 males (see Figure 5D). Of the genetic and chemical perturbations gene set collection, the gene set "MEISSNER NPC HCP with H3K4ME2 and H3K27ME3" was among the most significantly differentially regulated gene sets whose members were down-regulated in MORF1 males, along with other curated gene sets characterizing methylation patterns, suggesting a role for histone methylation and chromatin structure in the MORF1 response. Top gene candidates identified via differential expression (Cyp2c7) and GSEA (PPAR $y$ and Hnf1a) were also examined by qPCR to provide additional validation of sequencing results (Data S1; Figure 3).

\section{4 | DISCUSSION}

Opioid use among females of reproductive age remains at historically high levels, despite efforts to reduce prescribing rates. ${ }^{3}$ When considering opioid use in the context of reproductive outcomes, the primary focus is often on continuing use during pregnancy and effects on the fetus and newborn infant. Moreover, when potential long-term outcomes of fetal opioid exposure are hypothesized, they often focus on neurocognitive effects related to behavioral regulation. Yet effects of both exogenous and endogenous opioids on glucose regulation have been appreciated for decades. ${ }^{28}$ Indeed, increased risk for type 2 diabetes in breast cancer survivors correlates with cumulative opioid use $^{29}$ and increased prevalence of metabolic syndrome is reported in opioid dependent patients. ${ }^{30}$ The current findings, however, suggest that this increased risk is not limited to the female consuming these drugs but can also extend to future progeny.

Metabolic diseases, including obesity and diabetes, are major public health issues of growing concern. ${ }^{31}$ In the human population, parental and grandparental experiences (ie, stress and nutritional status), even those experiences occurring prior to conception, can predispose future progeny to metabolic disease. ${ }^{32,33}$ Thus, in addition to the effects of the fetal and postnatal environment, transgenerational epigenetic inheritance may also moderate the risk of metabolic disease. ${ }^{34,35}$ While parental exposures to drugs of abuse have been demonstrated to alter reward pathways in nonexposed offspring, ${ }^{36,37}$ the primary focus has been on substance use disorder. The current findings demonstrate that effects of maternal opioid use prior to pregnancy extend beyond substance abuse liability and include alterations in metabolic and neuroendocrine regulation with potential health consequences that are exacerbated in environments in which high fat and sugary diets are the norm.

In the present study, delayed sexual maturation in MORF1 males served as an early indicator of neuroendocrine disruption, even before any diet-induced effects were observed. It is possible that such neuroendocrine changes influenced subsequent physiological effects induced by HSD in MORF1 males. Moreover, initiating the HSD diet during pubertal development may have resulted in more profound effects on MORF1 males than had the diet been initiated in adulthood. Indeed, previous findings have observed profound effects on hypothalamic transcription in preclinical models that initiate a high sugar diet during adolescence but not in adulthood. ${ }^{38}$ In response to maintenance on FSD, MORF1 males went on to demonstrate increased bodyweight gain, fasting glucose, insulin, and corticosterone as well as increased ARC NPY. The higher bodyweights were not due to differences in locomotor activity. Rather, MORF1 males were generally hyperphagic under FSD conditions, an effect associated with elevated expression of NPY, a potent orexigen. Of note, the NPY inactive precursor, proNPY, was increased in MORF1 males irrespective of dietary condition. Such findings suggest that MORF1 males may be predisposed to metabolic dysfunction, displaying increased baseline 
expression of proNPY, with counter-regulatory post-translational mechanisms in place to suppress NPY production under CD but not once animals are metabolically challenged with FSD.

Similarly, POMC peptide was significantly increased in CD-maintained MORF1 males. One hypothesis is that this up-regulation could serve as a counter-regulatory homeostatic mechanism in a phenotype prone toward a positive energy balance. The effect on POMC was not observed under FSD conditions, suggesting that diet may challenge this regulatory mechanism in MORF1 males. Thus, under FSD conditions, these compensatory mechanisms are disrupted leading to a positive energy balance and glucose dyshomeostasis. As differences in POMC were observed under control conditions, we speculated that up-regulation of this anorexogenic peptide may play a role in the propagation of this phenotype. Indeed, previous studies demonstrate that fetal alcohol exposure induces hypermethylation of Pomc, and this epigenetic mark is maintained over three generations and is associated with later life adult-onset disease. ${ }^{39}$ Because hypomethylation of the distal Pomc promoter increases POMC expression, ${ }^{40}$ we assessed the Pomc locus for changes in methylation status under baseline conditions. However, DNA methylation within the Pomc locus was similar between SALF1 and MORF1 males. This suggests that the observed differences in POMC expression are regulated by other mechanisms.

To assess the functional effects of differential ARC POMC expression, $\alpha-M S H$ and $\beta$-EP levels were examined in PVN and VTA, where they are known to augment energy balance and metabolism via receptor-mediated stimulation of neuronal targets. ${ }^{41}$ Under the CD condition, MORF1 males had reduced levels of a-MSH within the PVN, even in the presence of increased ARC POMC expression. This effect was not observed under FSD conditions. Thus, findings suggest diminished melanocortin tone within the PVN of MORF1 males, and further support the notion that even under the $C D$ conditions, these animals are primed for a metabolically adverse phenotype. ${ }^{42}$ Within the VTA, no differences in a-MSH were observed, demonstrating region-specific modifications in POMC-derived neuropeptide distribution.

Under FSD conditions, MORF1 males display increased $\beta$-EP levels within the VTA, which may contribute to the observed increase in bodyweight, as $\beta$-EP signals within the VTA promote a positive energy balance. ${ }^{43}$ Moreover, $\beta$-EP reduces POMC activity via an autoregulatory mechanism ${ }^{44}$; thus, a $\beta$-EP mediated inhibition of POMC activity in MORF1 males may account for the absence of differences in ARC POMC expression in FSD-maintained MORF1 males. In addition, as subthreshold amounts of $\beta$-EP suppress the metabolic effects induced by $\alpha-\mathrm{MSH},{ }^{45}$ it is likely that FSD-fed MORF1 males may also have diminished melanocortin tone within the VTA. Notably, increasing melanocortin signaling within the VTA protects against the development of diet-induced obesity. ${ }^{46}$ Thus, altered $\alpha-\mathrm{MSH}$ and $\beta$-EP activity within the PVN and VTA is associated with the adverse MORF1 metabolic phenotype. Furthermore, elevated $\beta$-EP levels within the VTA suggest that the hedonic value of food in FSD MORF1 males may be elevated, resulting in increased caloric intake. Such an outcome may suggest significant differences in reward valence of palatable food as a consequence of maternal opioid history. We have previously measured self-administration of sucrose pellets in MORF1 males (fixed ratio and progressive ratio) and did not observes any significant differences when compared with SALF1 males ${ }^{47}$; however, it is possible that either the high fat component of the FSD or the continuous availability of this highly palatable food stimulated increased feeding behaviors in MORF1 males. Indeed, endogenous opioids have been demonstrated to influence the hedonic value of highly palatable food ${ }^{48}$ suggesting that dysregulation of opioid modulation of reward systems, similar to what we have observed in previous studies. ${ }^{11,13}$

Impairments in glucose homeostasis also emerged in FSD-MORF1 males. To examine more proximal mechanisms underlying MORF1 fasting hyperglycemia, insulin and CORT were also evaluated. While CD-F1 males display comparable fasting plasma insulin, FSD-MORF1 males demonstrate elevated insulin when compared with FSD-SALF1 males. Previous studies suggest, similar to our findings in FSD-SALF1 males, chronic exposure to high fat diets diminish the hyperinsulinemia that is observed when animals are first transitioned to a high fat diet. ${ }^{49}$ Such an effect suggests that the normal adaptations observed in response to maintenance on a FSD are modified based on maternal opioid history resulting in early the emergence of insulin resistance in FSD-MORF1 males, a pathologic condition that mediates the prediabetic/diabetic state. ${ }^{50}$ Future studies are needed to assess insulin sensitivity in central and peripheral tissues with respect to the role of insulin in facilitating MORF1 glucose dysregulation. Nonetheless, our current data reveals that MORF1 males are predisposed to the development of type 2 diabetes following only 6 weeks on an obesogenic diet. The metabolic profile of FSD-MORF1 males suggests an increased risk for the development of metabolic syndrome ${ }^{51}$; as such, it is expected that MORF1 males would be susceptible to the development of associated comorbidities (ie, cardiovascular disease and hypertension).

FSD-MORF1 males also displayed increased levels of CORT, which may contribute to the hyperglycemic state as elevated CORT levels are gluconeogenic. ${ }^{52}$ While elevated NPY likely plays a role in the hyperphagia demonstrated by FSD-maintained MORF1 males, NPY also positively regulates the HPA axis, thus increasing CORT output. ${ }^{53}$ Indeed, NPY, CORT, and insulin are regulated in tandem to affect multiple aspects of energy homeostasis. Thus, elevated levels of NPY may result in increased CORT. It is also possible that MORF1 males are more sensitive to activation of the HPA axis induced by fasting conditions, resulting in increased CORT, which may then increase NPY expression and gluconeogenesis despite elevated insulin levels. Importantly, these effects are only observed in MORF1 males following maintenance on the FSD, indicative of an interaction between maternal opioid history and diet condition on hypothalamic regulation of homeostatic processes critical for regulating both feeding behavior and glucose regulation.

To further provide an unbiased assessment of hypothalamic modifications related to both maternal opioid history and diet, transcriptional changes in the ARC were examined using RNAseq. Effects of maternal opioid history only reached significance in FSD-maintained F1 males. The overall pattern of these changes implicate down- 
regulation of genes associated with synaptic plasticity and neurotransmission coincident with up-regulation of immune, inflammatory, and metabolic-related genes. It should be noted that neither the POMC nor the NPY gene reached significance in our sequencing analysis, although we have previously observed significant up-regulation in the POMC gene in MORF1 males ${ }^{10,13}$ and protein expression was significantly impacted by both maternal opioid history and diet. Given the large number of genes probed via sequencing and the statistical corrections required for multiple comparisons, the failure to observe significant effects on relatively small changes in gene expression was not unexpected, particularly given that RNA sequencing was conducted under fasting conditions, which likely induced a robust transcriptional response. Indeed, it was for those reasons that we opted to focus our analysis on gene sets rather than on individual gene targets, as significant differences in homeostatic processes that develop over a protracted period of time in response to dietary intake likely result in subtle changes in gene expression across coordinated gene networks rather than having a large effect on single gene targets.

The use of gene set enrichment analyses confirm and extend these findings, identifying a number of pathways that may a play a role in the metabolic dyshomeostasis observed in FSD-maintained MORF1 males and which suggest additional risk for adult-onset disease under these conditions. For example, the peroxisome proliferator-activated receptor (PPAR) signaling pathway was significantly up-regulated in FSDMORF1 males. Interestingly, not only is this pathway implicated in nutritional regulation of metabolism ${ }^{54}$ but it is also a potential link between metabolic dysregulation and cancer. ${ }^{55}$ Up-regulation of one member of this pathway, PPAR $\gamma$, was significantly increased in FSDMORF1 males when measured using qPCR (Figure S3). Additionally, a number of transcription factors whose targets showed significant

\section{Methods}

Saline (SALF0) or Morphine (MORF0) Exposure Regimen
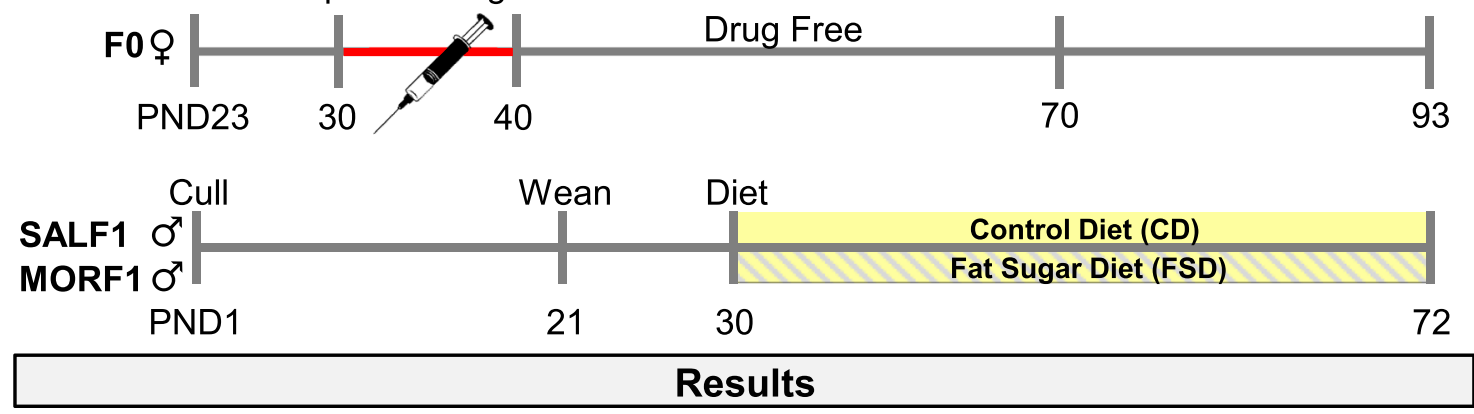

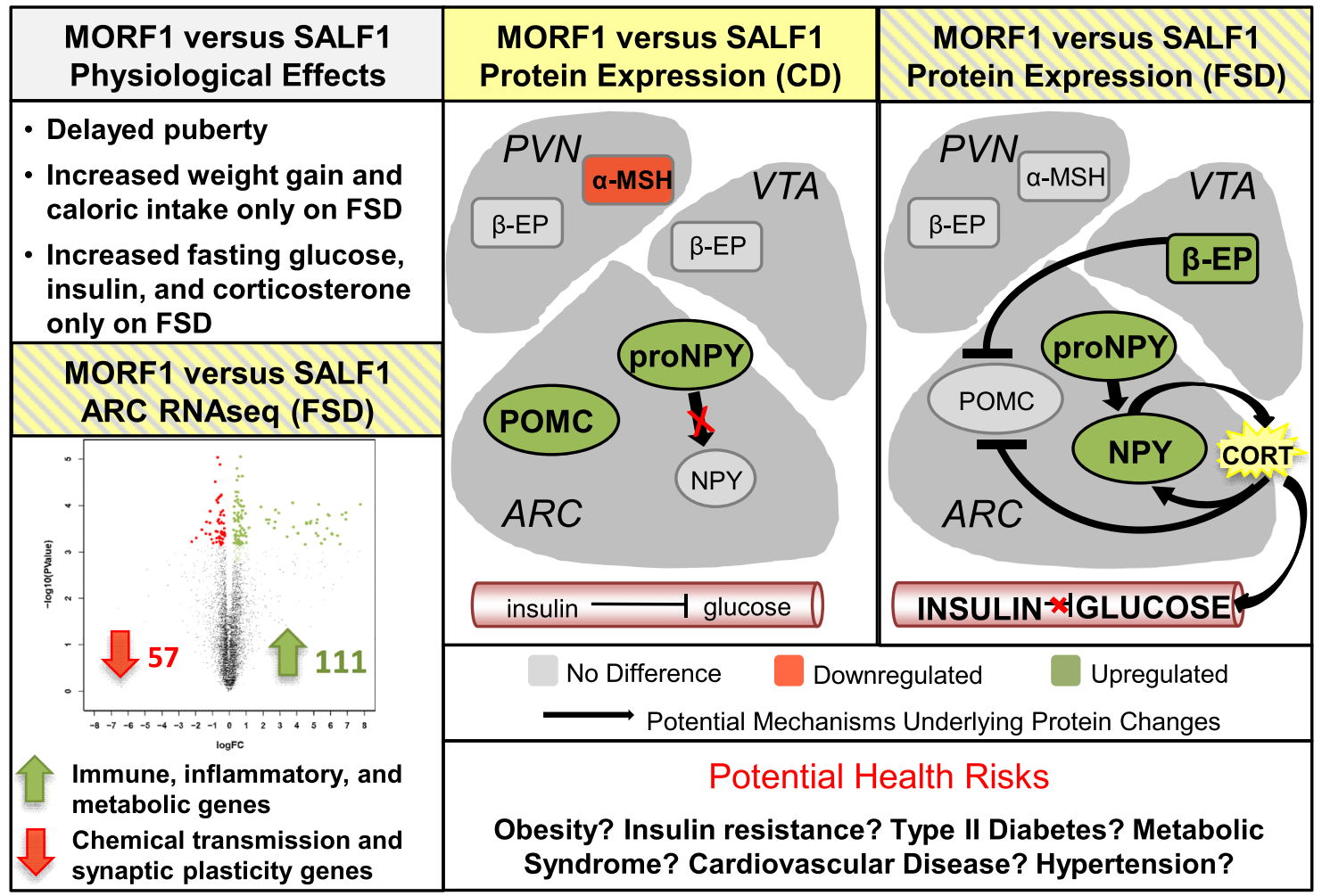

FIGURE 6 Schematic of findings, potential mechanisms, and potential risks 
dysregulation were identified using GSEA, including Hnf1, a transcription factor linked with mature onset diabetes of the young. ${ }^{56}$ Protein-protein interactions connecting the targets of the transcription factors modified in FSD-MORF1 males were also noted (Figure 5D), while other significant gene set enrichment implicates modifications in histone methylation within the hypothalamus of FSD-maintained MORF1 males. Thus, the impact on neurodevelopmental processes appears to involve multiple regulatory mechanisms, not only transcriptional regulation but also protein-protein interactions and chromatin accessibility. These analyses demonstrate the power of using gene sets to identify shifts in the transcriptional landscape, as opposed to simply examining differences in individual genes. Indeed, while dysregulation of the Hnf1a transcription factor was identified in FSD-MORF1 males, expression of Hnfla was only significantly up-regulated in MORF1 CD males when examined using qPCR (Figure S3). Overall, these data point to the capacity for maternal opioid exposure prior to conception to fundamentally alter transcriptional responses under particular dietary conditions in a brain region critical for homeostasis.

As summarized in Figure 6, these findings suggest that MORF1 males are innately primed for metabolic dyshomeostasis, which is revealed at an early age in response to a relatively brief ( 6 week) dietary challenge. Thus, a maternal history of opioid exposure interacts with F1 offspring diet to disturb hypothalamic and neuroendocrine systems that influence sexual maturation, energy metabolism, synaptic plasticity, and likely immune function mediated by profound changes in the arcuate nucleus of the hypothalamus. Many of the observed effects on gene transcription and metabolic function increase the risk that MORF1 males will develop other pathologies including other metabolic diseases (ie, cardiovascular disease), neurodevelopmental disorders, and potentially cancer.

We conclude that maternal opioid exposure prior to conception increases the risk that future male offspring will develop type 2 diabetes when maintained for a relatively brief period on a diet high in fat and sugar. These findings implicate maternal opioid exposure, even when use is discontinued prior to pregnancy, as a potential risk factor for the adult onset of metabolic dysfunction in the next generation.

\section{ACKNOWLEDGEMENTS}

This work was supported by the National Institutes of Health RO1 DA025674 (E.M.B.) and a Tufts Collaborates Award (E.M.B. and D.S.).

\section{CONFLICT OF INTEREST}

The authors declare no competing financial interest.

\section{AUTHORS CONTRIBUTION}

Study conception and design: EMB and FMV; Data acquisition AMT, FMV, SB, CM, FQ, DS, and EMB; Data analysis and interpretation: AMT, FMV, CMS, SB, CM, FQ, DS, and EMB; Manuscript drafting and revision: $\mathrm{AMT}, \mathrm{FMV}, \mathrm{CMS}, \mathrm{SB}, \mathrm{CM}, \mathrm{FQ}$, DS, and EMB.

\section{ORCID}

Anika M. Toorie (iD) https://orcid.org/0000-0002-7235-5519

Elizabeth M. Byrnes (1) https://orcid.org/0000-0002-8458-7712

\section{REFERENCES}

1. Kolodny A, Courtwright DT, Hwang CS, Kreiner P, Eadie JL, Clark TW, Alexander GC The prescription opioid and heroin crisis: a public health approach to an epidemic of addiction. Annu Rev Public Health 2015; 36 : 559-574. 2015/01 /13. https://doi.org/10.1146/annurev-publhealth031914-122957, 1.

2. National Institute on Drug Abuse and University of Michigan. Institute for Social Research. Monitoring the future, national survey results on drug use. NIH publication. Bethesda, Md.: National Institute on Drug Abuse, U.S. Dept. of Health and Human Services, National Institutes of Health, p. 1 online resource ( $\mathrm{v}$.

3. Ostling PS, Davidson KS, Anyama BO, Helander EM, Wyche MQ, Kaye AD America's opioid epidemic: a comprehensive review and look into the rising crisis. Curr Pain Headache Rep 2018; 22: 32. 2018/04 /06. https://doi.org/10.1007/s11916-018-0685-5, 5.

4. Bodnar RJ. Endogenous opiates and behavior: 2016. Peptides 2018; 101: 167-212. 2018/01/26. https://doi.org/10.1016/j.peptides 2018.01.011

5. Ramabadran K, Bansinath M. Glucose homeostasis and endogenous opioid peptides. Int J Clin Pharmacol Ther Toxicol 1990; 28: 89-98. 1990/03 /01.

6. Byrnes EM. Chronic morphine exposure during puberty induces longlasting changes in opioid-related mRNA expression in the mediobasal hypothalamus. Brain Res. 2008;1190:186-192. https://doi.org/10.101 6/j.brainres.2007.11.018

7. Byrnes JJ, Babb JA, Scanlan VF, Byrnes EM. Adolescent opioid exposure in female rats: transgenerational effects on morphine analgesia and anxiety-like behavior in adult offspring. Behav Brain Res. 2011;218(1):200-205. https://doi.org/10.1016/j.bbr.2010.11.059

8. Byrnes JJ, Johnson NL, Carini LM, Byrnes EM. Multigenerational effects of adolescent morphine exposure on dopamine D2 receptor function. Psychopharmacology (Berl). 2013;227(2):263-272. https:// doi.org/10.1007/s00213-012-2960-1

9. Johnson NL, Carini L, Schenk ME, Stewart M, Byrnes EM. Adolescent opiate exposure in the female rat induces subtle alterations in maternal care and transgenerational effects on play behavior. Front Psychiatry. 2011;2:29. https://doi.org/10.3389/fpsyt.2011.00029

10. Vassoler FM, Johnson-Collins NL, Carini LM, Byrnes EM Next generation effects of female adolescent morphine exposure: sex-specific alterations in response to acute morphine emerge before puberty. Behav Pharmacol 2014; 25: 173-181. 2014/02/25. https://doi.org/ 10.1097/FBP.0000000000000032, 2.

11. Vassoler FM, Wright SJ and Byrnes EM. Exposure to opiates in female adolescents alters mu opiate receptor expression and increases the rewarding effects of morphine in future offspring. Neuropharmacology 2016; 103: 112-121. 2015/12/25. 10.1016/j.neuropharm. 2015.11.026

12. Vassoler FM, Toorie AM and Byrnes EM. Transgenerational blunting of morphine-induced corticosterone secretion is associated with dysregulated gene expression in male offspring. Brain Res 2018; 1679: 19-25. 2017/11/14. 10.1016/j.brainres.2017.11.004

13. Vassoler FM, Toorie AM and Byrnes EM. Increased cocaine reward in offspring of females exposed to morphine during adolescence. Psychopharmacology (Berl) 2019; 236: 1261-1272. 2018/12/07. 10.1007/ s00213-018-5132-0 
14. Levin $\mathrm{BE}$, Routh $\mathrm{VH}$. Role of the brain in energy balance and obesity. Am J Physiol. 1996;271:R491-R500. Editorial. Research Support, U.S. Gov't, Non-P.H.S. Research Support, U.S. Gov't, P.H.S. Review 1996/ 09/01

15. Chhabra KH, Adams JM, Fagel B, et al. Hypothalamic POMC deficiency improves glucose tolerance despite insulin resistance by increasing glycosuria. Diabetes. 2016;65(3):660-672. https://doi.org/10.2337/db150804

16. Faulkner LD, Dowling AR, Stuart RC, Nillni EA, Hill JW. Reduced melanocortin production causes sexual dysfunction in male mice with POMC neuronal insulin and leptin insensitivity. Endocrinology. 2015;156(4):1372-1385. https://doi.org/10.1210/en.2014-1788

17. Candler T, Kuhnen P, Prentice AM, Silver M. Epigenetic regulation of POMC; implications for nutritional programming, obesity and metabolic disease. Front Neuroendocrinol 2019: 100773. 2019/07/26. https://doi.org/10.1016/j.yfrne.2019.100773, 54.

18. Raubenheimer PJ, Young EA, Andrew R, Seckl JR The role of corticosterone in human hypothalamic-pituitary-adrenal axis feedback. Clin Endocrinol (Oxf) 2006; 65: 22-26. 2006/07/05. https://doi.org/ 10.1111/j.1365-2265.2006.02540.x, 1.

19. Spear LP. Adolescent brain development and animal models. Ann N Y Acad Sci. 2004;1021:23-26. https://doi.org/10.1196/annals.1308.002

20. Korenbrot CC, Huhtaniemi IT, Weiner RI. Preputial separation as an external sign of pubertal development in the male rat. Biol Reprod. 1977;17(2):298-303.

21. Dobin A, Davis CA, Schlesinger F, Drenkow J, Zaleski C, Jha S, Batut P, Chaisson M, Gingeras TR STAR: ultrafast universal RNA-seq aligner. Bioinformatics 2013; 29: 15-21. 2012/10/30. https://doi.org/ 10.1093/bioinformatics/bts635, 1.

22. Robinson $M D$ and Oshlack $A$. A scaling normalization method for differential expression analysis of RNA-seq data. Genome Biol 2010; 11: R25. 2010/03/04. 10.1186/gb-2010-11-3-r25

23. Wei J, Carroll RJ, Harden KK, Wu G. Comparisons of treatment means when factors do not interact in two-factorial studies. Amino Acids 2012; 42: 2031-2035. 2011/05/07. https://doi.org/10.1007/ s00726-011-0924-0

24. Robinson MD, McCarthy DJ and Smyth GK. edgeR: a bioconductor package for differential expression analysis of digital gene expression data. Bioinformatics 2010; 26: 139-140. 2009/11/17. 10.1093/bioinformatics/btp616

25. Yu G, Wang LG, Han Y, He QY clusterProfiler: an R package for comparing biological themes among gene clusters. OMICS 2012; 16: 284-287. 2012/03/30. https://doi.org/10.1089/omi.2011.0118, 5.

26. Subramanian A, Tamayo P, Mootha VK, Mukherjee S, Ebert BL, Gillette MA, Paulovich A, Pomeroy SL, Golub TR, Lander ES, Mesirov JP Gene set enrichment analysis: a knowledge-based approach for interpreting genome-wide expression profiles. Proc Natl Acad Sci U S A 2005; 102: 15545-15550. 2005/10/04. https://doi.org/10.1073/ pnas.0506580102, 43 .

27. Szklarczyk D, Franceschini A, Wyder S, Forslund K, Heller D, Huerta-Cepas J, Simonovic M, Roth A, Santos A, Tsafou KP, Kuhn M, Bork P, Jensen $L$, Von Mering C STRING v10: protein-protein interaction networks, integrated over the tree of life. Nucleic Acids Res 2015; 43: D447-452. 2014/10/30. https://doi.org/10.1093/nar/gku1003, D1.

28. Lala A, Bouloux P, Tamburrano G, Gale E Opioid peptides and glucose metabolism. J Endocrinol Invest 1987; 10: 95-104. 1987/02/01. https://doi.org/10.1007/BF03347164, 1.

29. Yang SP, Muo CH, Wang IK, Chang YJ, Lai SW, Lee CWS, Morisky DE Risk of type 2 diabetes mellitus in female breast cancer patients treated with morphine: a retrospective population-based time- dependent cohort study. Diabetes Res Clin Pract 2015; 110: 285-290. 2015/10/31. https://doi.org/10.1016/j.diabres.2015.10.005, 3.

30. Mattoo SK, Chakraborty K, Basu D, Ghosh A, Vijaya Kumar KG, Kulhara P Prevalence \& correlates of metabolic syndrome in alcohol \& opioid dependent inpatients. Indian J Med Res 2011;134:341-348. 2011/10/12.

31. Tanner RM, Brown TM, Muntner P. Epidemiology of obesity, the metabolic syndrome, and chronic kidney disease. Curr Hypertens Rep. 2012;14(2):152-159. https://doi.org/10.1007/s11906-0120254-y

32. Hales CN and Barker DJ. Type 2 (non-insulin-dependent) diabetes mellitus: the thrifty phenotype hypothesis. 1992. Int J Epidemiol 2013;42:1215-1222. https://doi.org/10.1093/ije/dyt133, 5.

33. Hanafi MY, Saleh MM, Saad MI, Abdelkhalek TM, Kamel MA. Transgenerational effects of obesity and malnourishment on diabetes risk in F2 generation. Mol Cell Biochem. 2016;412(1-2):269-280. https://doi.org/10.1007/s11010-015-2633-6

34. Wei $\mathrm{Y}$, Schatten $\mathrm{H}$, Sun QY. Environmental epigenetic inheritance through gametes and implications for human reproduction. Human reproduction update. 2015;21(2):194-208. https://doi.org/10.1093/ humupd/dmu061

35. Mathes WF, Kelly SA, Pomp D. Advances in comparative genetics: influence of genetics on obesity. Br J Nutr. 2011;106(Suppl 1): S1-S10. Research Support, N.I.H., Extramural Review 2011/10/26. 10.1017/S0007114511001905

36. Vassoler FM, Byrnes EM and Pierce RC. The impact of exposure to addictive drugs on future generations: physiological and behavioral effects. Neuropharmacology 2014;76 Pt B:269-275. 10.1016/j. neuropharm.2013.06.016

37. Vassoler FM, Sadri-Vakili G. Mechanisms of transgenerational inheritance of addictive-like behaviors. Neuroscience. 2014;264:198-206. https://doi.org/10.1016/j.neuroscience.2013.07.064

38. Harrell CS, Burgado J, Kelly SD, Johnson ZP, Neigh GN. High-fructose diet during periadolescent development increases depressive-like behavior and remodels the hypothalamic transcriptome in male rats. Psychoneuroendocrinology. 2015;62:252-264.

39. Sarkar DK. Male germline transmits fetal alcohol epigenetic marks for multiple generations: a review. Addict Biol 2016; 21: 23-34. 2015/ 01/13. https://doi.org/10.1111/adb.12186

40. Wu Y, Patchev AV, Daniel G, Almeida OF, Spengler D. Early-life stress reduces DNA methylation of the Pomc gene in male mice. Endocrinology. 2014;155(5):1751-1762. https://doi.org/10.1210/ en.2013-1868

41. Solomon S. POMC-derived peptides and their biological action. Ann N Y Acad Sci. 1999;885:22-40.

42. Luchtman DW, Chee MJ, Doslikova B, Marks DL, Baracos VE, Colmers WF. Defense of elevated body weight setpoint in dietinduced obese rats on low energy diet is mediated by loss of melanocortin sensitivity in the paraventricular hypothalamic nucleus. PLoS One. 2015;10(10):e0139462. https://doi.org/10.1371/journal. pone. 0139462

43. Noel MB, Wise RA. Ventral tegmental injections of morphine but not U-50,488 H enhance feeding in food-deprived rats. Brain Res. 1993;632(1-2):68-73.

44. Pennock RL, Hentges ST. Differential expression and sensitivity of presynaptic and postsynaptic opioid receptors regulating hypothalamic proopiomelanocortin neurons. J Neurosci. 2011;31(1):281-288. https://doi.org/10.1523/JNEUROSCI.4654-10.2011

45. Dutia R, Meece K, Dighe S, Kim AJ, Wardlaw SL. beta-Endorphin antagonizes the effects of alpha-MSH on food intake and body weight. 
Endocrinology. 2012;153(9):4246-4255. https://doi.org/10.1210/ en.2012-1166

46. Andino LM, Ryder DJ, Shapiro A, et al. POMC overexpression in the ventral tegmental area ameliorates dietary obesity. The Journal of endocrinology. 2011;210(2):199-207. https://doi.org/10.1530/JOE-100418

47. Vassoler FM, Oliver DJ, Wyse C, Blau A, Shtutman M, Turner JR, Byrnes EM Transgenerational attenuation of opioid self-administration as a consequence of adolescent morphine exposure. Neuropharmacology 2017;113:271-280. 2016/11/05. https://doi.org/10.1016/j. neuropharm.2016.10.006, Pt A.

48. Will MJ, Franzblau EB and Kelley AE. Nucleus accumbens mu-opioids regulate intake of a high-fat diet via activation of a distributed brain network. J Neurosci 2003;23:2882-2888. 2003/04/10.

49. Cruciani-Guglielmacci C, Vincent-Lamon M, Rouch C, Orosco M., Ktorza A., Magnan C. Early changes in insulin secretion and action induced by high-fat diet are related to a decreased sympathetic tone. Am J Physiol Endocrinol Metab 2005; 288: E148-154. 2004/09/09. https://doi.org/10.1152/ajpendo.00225.2004, 1.

50. Robertson RP, Olson LK, Zhang HJ. Differentiating glucose toxicity from glucose desensitization: a new message from the insulin gene. Diabetes. 1994;43:1085-1089.

51. Abdul-Ghani MA, Matsuda M, Jani R, et al. The relationship between fasting hyperglycemia and insulin secretion in subjects with normal or impaired glucose tolerance. Am J Physiol Endocrinol Metab. 2008;295 (2):E401-E406. https://doi.org/10.1152/ajpendo.00674.2007

52. Jimeno B, Hau M and Verhulst S. Corticosterone levels reflect variation in metabolic rate, independent of 'stress'. Sci Rep 2018; 8: 13020. 2018/08/31. https://doi.org/10.1038/s41598-018-31258-z.
53. Sainsbury A, Rohner-Jeanrenaud F, Cusin I, et al. Chronic central neuropeptide $\mathrm{Y}$ infusion in normal rats: status of the hypothalamopituitary-adrenal axis, and vagal mediation of hyperinsulinaemia. Diabetologia. 1997;40(11):1269-1277. https://doi.org/10.1007/ s001250050820

54. Diano S, Liu ZW, Jeong JK, et al. Peroxisome proliferation-associated control of reactive oxygen species sets melanocortin tone and feeding in diet-induced obesity. Nature medicine. 2011;17(9):1121-1127. https://doi.org/10.1038/nm.2421

55. Fanale D, Amodeo V, Caruso S. The interplay between metabolism, PPAR signaling pathway, and cancer. PPAR Research. 2017;2017:1830626. https://doi.org/10.1155/2017/1830626

56. Najmi LA, Aukrust I, Flannick J, et al. Functional investigations of HNF1A identify rare variants as risk factors for type 2 diabetes in the general population. Diabetes. 2017;66(2):335-346. https://doi. org/10.2337/db16-0460

\section{SUPPORTING INFORMATION}

Additional supporting information may be found online in the Supporting Information section at the end of the article.

How to cite this article: Toorie AM, Vassoler FM, Qu F, et al. A history of opioid exposure in females increases the risk of metabolic disorders in their future male offspring. Addiction Biology. 2019;e12856. https://doi.org/10.1111/adb.12856 